\title{
Understanding the Postgraduate Education Market for Better Marketing and Decision Making: A Clustering Analysis
}

\author{
Ali Marstawi \\ Faculty of Computer Science and Information Technology \\ Universiti Putra Malaysia \\ 43400 UPM Serdang, Selangor, Malaysia
}

\author{
Aida Mustapha \\ Faculty of Computer Science and Information Technology \\ Universiti Putra Malaysia \\ 43400 UPM Serdang, Selangor, Malaysia
}

\begin{abstract}
Enhancing the educational corporations is truly challenging mission due to the highly competitive nature of the business. Currently, there is emerging development within organizations to capitalize on their internal resources. This paper is taking data mining approach to strategize marketing for postgraduate studies by means of cluster analysis. The experiments were carried out using Oracle Data Miner tool, results are analyzed and discussed.
\end{abstract}

\section{General Terms:}

Educational Data Mining

\section{Keywords:}

Oracle Data Miner, Statistical Analysis, $K$-means Clustering

\section{INTRODUCTION AND RELATED WORK}

Because higher education is an essential factor for developing the society, it is imperative for learning institutions to invest time and resources in marketing the educational programs. As the number of universities increases dramatically, a very competitive environment has emerged among all the institutions to attract potential students. The main challenge remains on how to make use existing local resources, the very least the student application database.

Studies accomplished in the field of educational data mining present numerous case studies attempting to improve the educational experience according to the organization's perspective. The data mining approach in general begins by analyzing the available data and choosing the appropriate methods to extract the hidden patterns that is potentially useful to develop that organization.

Work by Romero and Ventura [6] present extensive review on educational data mining research efforts in the span of ten years; between 1995 and 2005. The same team [7] has also conducted a survey about the relevant studies carried out in the educational field relating the types of users and types of educational data provided. The data mining approach in [8] focus on three different predictive analysis, which are the neural network, logistic regression, and decision tree. Other algorithms in data approach include $K$-means clustering [1], association rules mining [4], as well as decision tree [3]. Overall, the research attempts to to predict student enrollment, to discover factors that impact the academic results among students or to analyze students characteristics based on their preference choice during registration at the university.

This research investigates the data from Universiti Putra Malaysia (UPM) regarding the postgraduate applicants, which includes Master and Ph.D candidates. The applicant database consists demographic information such as age, citizenship, gender, marital status; as well as field of study or domain-related information such as program applied and program offered. Clustering analysis is performed together with statistical methods for comparison purposes. The goal is to understand characteristics of the program applicants and distribution of the data, in effort to offer better education quality, better long-term investment plans, and more efficient targeted marketing.

The remainder of this paper proceeds as follows. Section 2 presents the cluster analysis for postgraduate education market, Section 3 details out the experimental setup, Section 4 discusses the results, and finally Section 5 concludes with some indication for future research.

\section{CLUSTER ANALYSIS IN POSTGRADUATE EDUCATION MARKET}

The objective of this paper is two-fold. First is to build a descriptive statistical model that describes the attributes that are most important to the university management and the relationships between one attribute with regards to the other attributes. Second is to use the descriptive data from the statistical model to perform clustering analysis in effort to help the university management for the same matter.

In data mining, the process of grouping a set of physical or abstract objects into classes of similar objects is called clustering. A cluster is a collection of data objects that are similar to one another within the same cluster and are dissimilar to the objects in other clusters. A cluster of data objects can be treated collectively as one group and so may be considered as a form of data compression [2]. 
The most commonly discussed distinction among different types of clustering is whether the set of clusters is nested or un-nested, or in more traditional terminology, whether it is hierarchical or partitional. Partitional clustering divides the set of data objects into non-overlapping subsets (clusters) such that each data object is in exactly one subset. If the clusters are permitted to have subclusters, then it is essentially a hierarchical clustering, whereby a set of nested clusters are organized as a tree. This research employs two clustering algorithms based on partitioning and hierarchical methods.

\subsection{Dataset}

The dataset under study is sourced from postgraduate applicant database in Universiti Putra Malaysia (UPM). Table 1 shows the selected attributes from the database under investigation.

\begin{tabular}{l}
$\begin{array}{c}\text { Table 1. The applicant } \\
\text { table. }\end{array}$ \\
\hline Selected Attributes \\
\hline ID \\
BUMISTATUS \\
CITIZEN_ID \\
LEVEL_OF_STUDY \\
CITIZENSHIP_STATUS \\
CHOICE1_APPLIED_ID \\
PROGRAM_OFFER_ID \\
DOMAIN \\
RELIGION_ID \\
GENDER_ID \\
MARITALSTATUS \\
AGE_APPLIED \\
FACULTY_ID
\end{tabular}

Based on Table 1 the attribute BUMISTATUS identifies whether the applicant is a bumiputra or otherwise and it has two distinct values. CITIZEN_ID identifies the nationality of the applicant, which has 246 values representing worldwide countries. LEVEL_OF_STUDY identifies the level of applicant and it has two values indicating Masters or Ph.D CITIZENSHIP_STATUS represents the locality of the applicant, whether the applicant is Malaysian or otherwise.

CHOICE1_APPLIED_ID indicates the program applied by the applicant. PROGRAM_OFFER_ID indicates the program offered by the university. DOMAIN represents the general domain of study with four different values. RELIGION_ID, GENDER, MARITALSTATUS and AGE_APPLIED attributes are self-explanatory. Next, the applicant and program tables were joined together to acquire the attribute FACULTY_ID which, as the name suggests, lists all the faculties available.

\subsection{Data Preprocessing}

Because data existing in real world are often noisy and most likely contain errors or outliers, pre-processing is imperative to treat any incomplete data such as missing or 'null' values, smoothing the noisy data, and removing or substituting the outliers. Table 2 shows the treatment to missing values in the applicant database.

To prepare for the final data, further pre-processing works were carried out. Since the applicant table contains data on all levels wise, postgraduate applicant was selected. A total of $0.5 \%$ from the attribute AGE_APPLIED that contains outliers (225 values from
-1977 to 15 and 60 values greater than 75) was discarded. Similar action was carried out with the attribute CHOICE1_APPLIED that contains values ranging from 1 to 1559 . However, only 50 records were found with a value greater than 302 . As the consequence, attributes with value greater than 302 were removed, the attribute GENDER and RELIGION are nominal attributes, therefore the missing values were replaced with mode.

Table 2. Treatment of missing values.

\begin{tabular}{|c|c|c|}
\hline Attribute & Type & $\begin{array}{l}\text { Replace missing } \\
\text { values by }\end{array}$ \\
\hline BUMISTATUS & Nominal & Mode \\
\hline CITIZEN_ID & Ordinal & Mode \\
\hline LEVEL_OF_STUDY & Nominal & Mode \\
\hline CHOICE1_APPLIED & Ordinal & Mode \\
\hline PROGRAM_OFFER & Ordinal & Mode \\
\hline DOMAIN & Ordinal & Mode \\
\hline GENDER_ID & Nominal & Mode \\
\hline MARITALSTATUS_ID & Nominal & Mode \\
\hline AGE_APPLIED & Ordinal & Average \\
\hline
\end{tabular}

In this work, the attribute AGE_APPLIED was discretized using the binning method because the attribute values range from 17 to 70 . However, all the values sit in different groups within the attribute range so that it can be divided equally. The ultimate advantage is that we were able to analyze AGE_APPLIED with a fewer values (equals to the bins number only) instead of dealing with it individually (all values from 17 to 70 ). Table 3 shows the sample data after pre-processing.

Table 3. Sample data from the applicant table.

\begin{tabular}{|c|c|c|c|c|c|c|c|c|c|c|c|}
\hline$\theta$ & 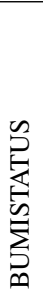 & 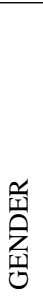 & 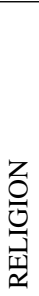 & 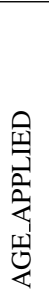 & 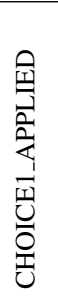 & 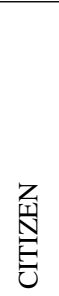 & 芳 & 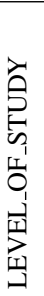 & 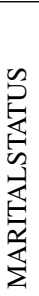 & 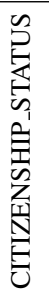 & 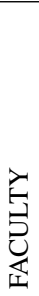 \\
\hline 3 & 2 & 1 & 7 & 23 & 192 & 96 & 2 & 3 & 3 & 6 & 12 \\
\hline 4 & 2 & 2 & 2 & 27 & 67 & 124 & 2 & 4 & 3 & 6 & 3 \\
\hline 7 & 2 & 1 & 2 & 28 & 289 & 124 & 2 & 3 & 3 & 6 & 15 \\
\hline 11 & 2 & 2 & 2 & 27 & 192 & 124 & 2 & 3 & 2 & 6 & 12 \\
\hline 13 & 2 & 1 & 2 & 23 & 238 & 233 & 2 & 4 & 3 & 6 & 13 \\
\hline 16 & 2 & 1 & 1 & 26 & 271 & 225 & 2 & 3 & 3 & 6 & 11 \\
\hline
\end{tabular}

\subsection{Cluster Analysis Experiment}

To perform the clustering experiment, the Oracle data miner [5] software tool was employed. Oracle data miner is powerful and easy tool, it provides a complete set of functions for cleaning and analyzing the data including efficient data mining algorithms and statistical functions. This tool is also preferred because the preprocessing database operations were run directly from the original database, which is an Oracle database. Figure 1 shows a snapshot of the tool showing the produced model.

The experiment was divided into two parts. In the first part, a comprehensive statistical analysis regarding the applicants' attributes was produced. The analysis covers a general statistic that 


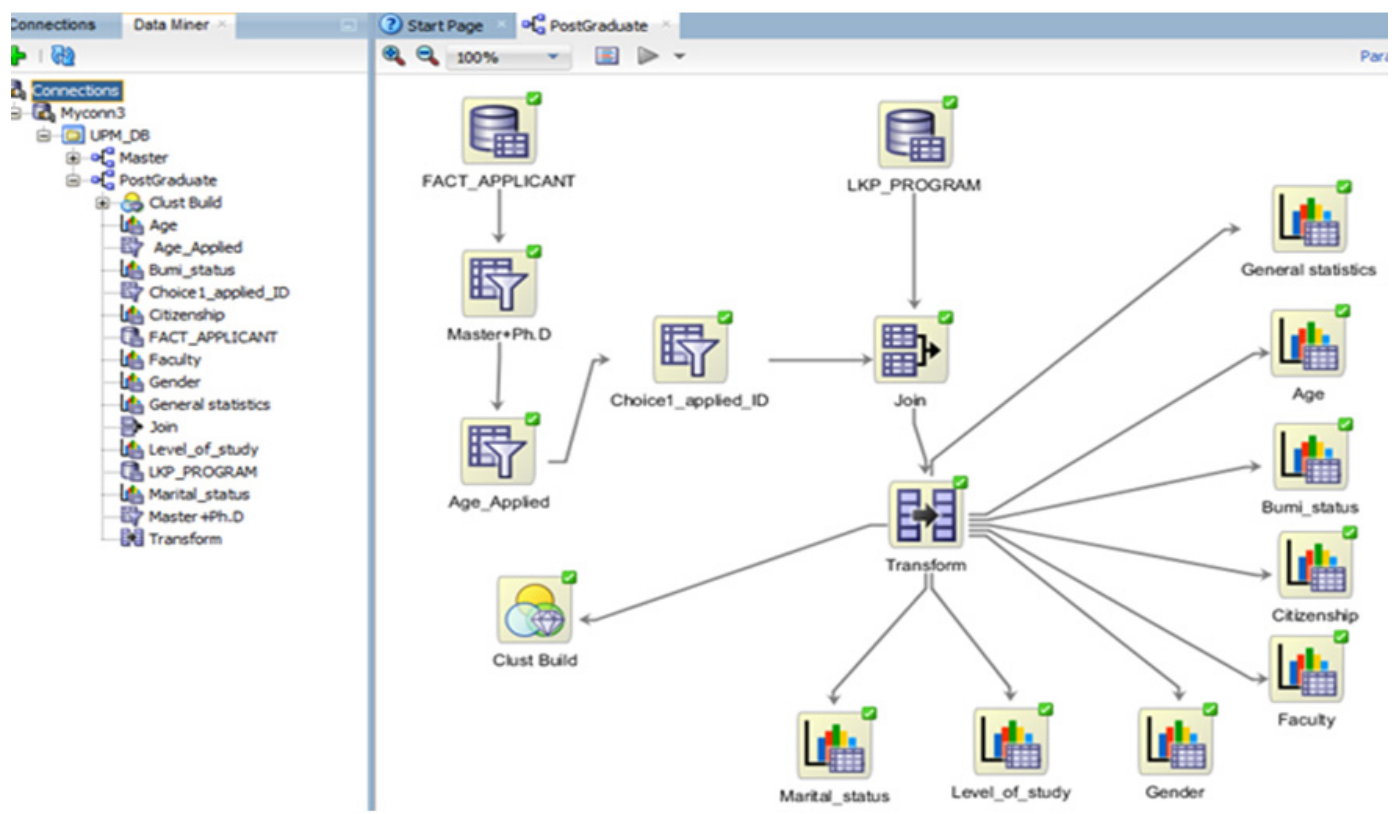

Fig. 1. Workflow in the Oracle data miner.

is not focused to any attribute, and multiple statistics carried out with respect to one particular attribute at a time. In the second part, a cluster analysis was performed using two clustering algorithms, which are partitional clustering and hierarchical clustering. Next, the results produced by each algorithm were compared.

From the experiment, it is found that the best number of clusters is $k=5$, as smaller number of $k$ resulted in smaller number of clusters, which would potentially hide some knowledge while the avoiding the cluster from too detail at the same time.

\section{RESULTS AND DISCUSSIONS}

\subsection{General Statistics}

The general statistics performed on the postgraduate UPM applicants revealed the following:

-AGE_APPLIED: The dominant age groups are $21.3<=25.6$, $25.6<=29.9,29.9<=34.2$ with values of $35.3 \%, 23.2 \%$ and $17.6 \%$ respectively.

-BUMISTATUS: This attribute splits between $54.8 \%$ for applicants who are bumiputra and $45.2 \%$ for non-bumiputra.

-CITIZENSHIP: This attribute is divided into $62.4 \%$ for local and $37.6 \%$ for foreigner.

-DOMAIN : This attribute has one outstanding value of $86 \%$ which refers to the Science domain.

- GENDER: This simple attribute splits into 52\% females and $48 \%$ males.

-LEVEL_OF_STUDY: This attribute splits into $79 \%$ for master applicants and $21 \%$ for Ph.D.

-MARITALSTATUS : $59.1 \%$ of the applicants for Masters and Ph.D level are singles and $40.4 \%$ are married.

-RELIGION: This attribute shows that $72 \%$ of the applicants are Muslim, while $7 \%$ are Buddha and the remaining 9\% are from other religions.
Table 4. The most applied programs.

\begin{tabular}{ccl}
\hline \multicolumn{3}{c}{ Applicants' Choice Statistics } \\
\hline Most applied program in general & Master of Business Administration \\
\hline \multirow{2}{*}{ Local } & Male & Master of Business Administration \\
\cline { 2 - 3 } & Female & Master of Education \\
\hline \multirow{2}{*}{ Foreigners } & Master of Science \\
\hline \multirow{3}{*}{ Age } & $<21$ & Master of Science \\
\cline { 2 - 3 } & {$[21,30]$} & Master of Business Administration \\
\cline { 2 - 3 } & {$[30,47]$} & Master of Education \\
\hline \multirow{2}{*}{ Level of Study } & $>47$ & Ph.D \\
\cline { 2 - 3 } & Master & Master of Business Administration \\
\cline { 2 - 3 } & Ph.D & -No dominant program- \\
\hline
\end{tabular}

-CHOICE_APPLIED_ID: Table 4 and Table 5 provide statistics about the applicant's applied program.

\subsection{Attribute-related Statistics}

Statistical analysis with regards to the attribute AGE:

-CITIZENSHIP: In this attribute, there is one prominent group for each value; for locals the prominent group is $(21<=25)$ with a value of $27 \%$, while for foreigners the prominent group is $(25<=29)$ with a value of $11.8 \%$.

-GENDER: A variance between the age groups found in this attribute; as the age group of $(21<=25)$ is dominant for females over males, the age group of $(29<=34)$ and above is dominant for males over females.

Statistical analysis with regards to the attribute BUMI_STATUS: 
Table 5. The most applied programs in terms of faculties.

\begin{tabular}{ll}
\hline \multicolumn{2}{c}{ Applicants' Choices Statistics } \\
\hline Faculty: & Program: \\
Faculty of Engineering & Master of Science 11,205 \\
Faculty of Educational Studies & Master of Education 10,250 \\
Graduate School of Management & Master of Business Administration 7,064 \\
Faculty of Modern Languages and Communication & Master of Corporate Communication 5,677 \\
Faculty of Computer Science and Information Technology & Master of Computer Science 4,318 \\
\hline
\end{tabular}

-AGE: The youngest age group $(21<=25)$ consists of more bumiputra; while other age groups consists of more nonbumiputra.

-CITIZENSHIP : Statistics regarding this attribute shows that $45 \%$ of the bumiputra applicants are local; while $17 \%$ foreigners.

-GENDER: There are more male and female bumiputra as compared to non-bumiputra.

-LEVEL_OF_STUDY: Statistics for this attribute shows that equal distribution for bumiputra and non-bumiputra as Masters applicants, but more bumiputra $\mathrm{Ph} . \mathrm{D}$ applicants as compared to non-bumiputra.

Statistical analysis with regards to the attribute CITIZENSHIP:

-AGE: Statistics shows that $27 \%$ of the local applicants are in the age range of $(21<=25)$, while $8 \%$ of the the applicants within the same age range are foreigners. For the age range between 25 and 38 , there is equivalent spread between local and foreigner. However, for applicants of older age, they are only local.

-FACULTY: It may be derived from this attribute that both local and foreigners are interested the most in the Faculty of Engineering and Educational Studies, whereby the split is $24.8 \%$ local applicants and $14.7 \%$ foreigners. The second most popular faculty consists of Faculty of Computer Science and Information Technology as well as Modern Languages and Communication with frequencies of $10.8 \%$ and $5.9 \%$ for local and foreigners, respectively

-GENDER: The applicants are composed of $22.9 \%$ local males and $25 \%$ foreigner males; while $39.6 \%$ local females and $12.5 \%$ foreigner females.

-LEVEL_OF_STUDY: Statistics for this attribute shows that $55.6 \%$ of Master applicants are local while $23.4 \%$ are foreigners. As for $\mathrm{Ph} . \mathrm{D}$ applicants it is $6.8 \%$ locals and $14.2 \%$ foreigners.

Statistical analysis with regards to the attribute GENDER:

-AGE: Females are generally older than males, but it is most obvious in the age between 21 and 25 there are $23.3 \%$ females and only $12.5 \%$ males. For the subsequent groups of age, the distribution is equal.

-LEVEL_OF_STUDY : It may be deduced that Master applicants are $35.2 \%$ males and $43.8 \%$ females, while for Ph.D $12.7 \%$ of the applicants males and $8.3 \%$ are females.

\subsection{Cluster Analysis}

Figure 2 shows the cluster analysis by applying the $K$-means clustering algorithm. The resulting $K$-means cluster group the applicants into the following categories. The first and dominant group is the group of single local females who are bumiputra their age ranges between 21 to 30 years. This group forms a percentage of $30 \%$ from the total applicants. The second is the group of single applicants who are non-bumiputra, age between 25 to 47 years with a percentage of $26 \%$. Next comes a unique group which consists of a group of all $\mathrm{Ph} . \mathrm{D}$ applicants who are non-bumiputra, foreigners and their age are between 25 to 47 years old. This group composed around $9 \%$ from the entire data. The last two clusters are actually concerning married applicants; local bumiputra between the age of 25 to 47 years $(21 \%)$ and the other one is for non-bumiputra foreigners, which constitues $9 \%$ from the total applicants.

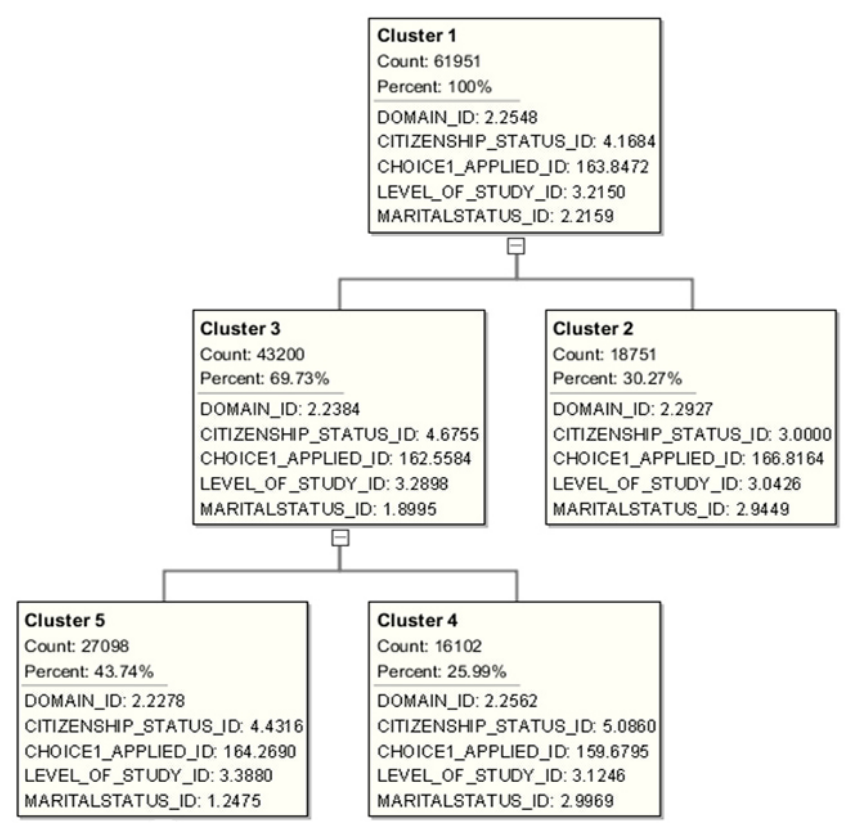

Fig. 2. Cluster produced by $K$-Means partitional clustering.

Figure 3 shows the cluster analysis by applying the $O$-cluster clustering algorithm. Note that the resulting $O$-cluster are totally different, in fact, while the first algorithm concentrated on residence and gender attributes, the $O$-cluster discards these attributes and focus mainly on the faculty and age attributes.

The first cluster comes very accurate, containing all the applicants which corresponds to the management domain and belong to the Graduate School of Management, while their age ranges between 21 to 42 years $(15 \%)$. The next group specifies all the Masters applicants who belong to the Faculty of Modern Languages and Communication as well as the Faculty of Computer Science and Information Technology are aged between 21 to 42 years (22\%)

The third cluster reveals the group of married applicants whose age are between 21 and 47 years and belong to the Faculty of Engineering, Science and Educational Studies. This group constitutes $25 \%$ from the total applicants. Finally, the the last two 
groups have two common features; both of these groups are single and their age are between 21 to 34 years old. The discrimination comes in the first group as applicants in this group are local and belong to the Faculty of Science, Economics and Management, and Forestry (7\%), while the second group belong to different variety of faculties regardless the citizenship status. These faculties include the Faculty of Engineering, Educational Study, and Agriculture $(29 \%)$.

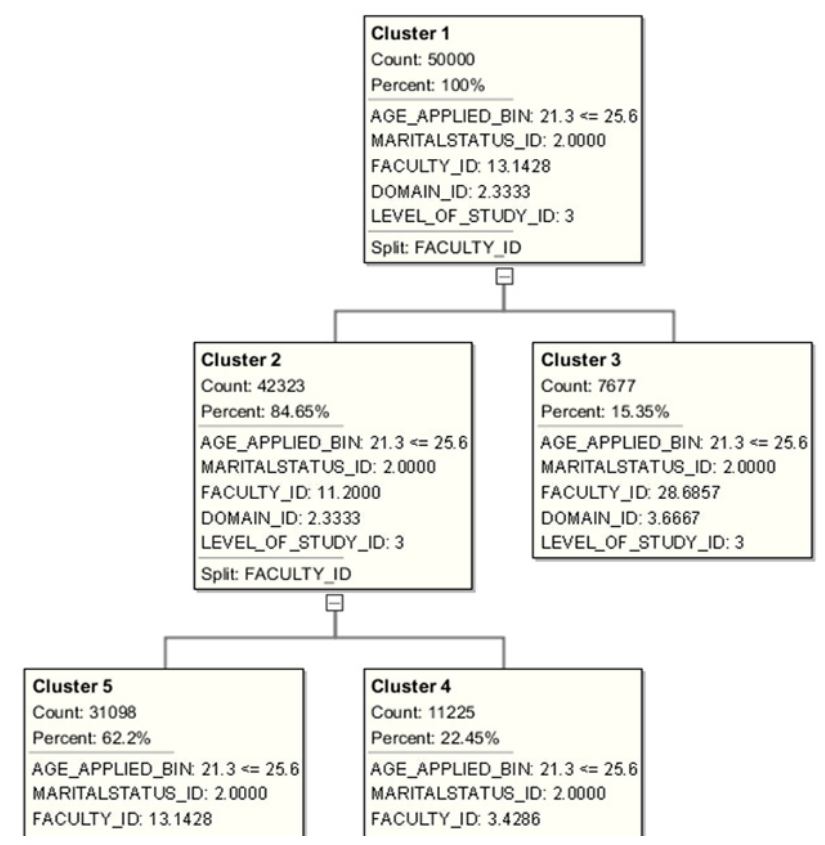

Fig. 3. Cluster produced by $O$-Cluster hierarchical clustering.

\section{CONCLUSION}

The paper presented statistical and cluster analysis on postgraduate application database in Universiti Putra Malaysia (UPM) for better marketing and decision making. The research is hoped to shed insights in how data mining could be used in understanding the postgraduate education market. In the future, the plan is to integrate the analysis into executive information system for strategic planning at the university level.

\section{REFERENCES}

[1] S. Ayesha, T. Mustafa, A.R. Sattar, and M.I. Khan. Data mining model for higher education system. Europen Journal of Scientific Research, 43(1):24-29, 2010.

[2] J. Han, M. Kamber, and J. Pei. Data Mining Concepts and Techniques. Morgan Kaufman, San Francisco, 3rd edition, 2006.

[3] M.B. Jasser, F. Sidi, A. Mustapha, and A.K. Binhamid. Mining students' characteristics and effects on university preference choice: A case study of applied marketing in higher education. International Journal of Computer Applications, 67(21):1-5, 2013.

[4] V. Kumar and A. Chadha. Mining association rules in student's assessment data. International Journal of Computer Science Issues, 9(5), 2012.

[5] Oracle. Odm: Oracle data miner. http://www.oracle.com/technetwork/database/options/advancedanalytics/odm/index.html.

[6] C. Romero and S. Ventura. Educational data mining: A survey from 1995 to 2005. Expert Systems with Applications, 33:135146, 2007.

[7] C. Romero and S. Ventura. Educational data mining: A review of the state of the art. IEEE Transactions on Systems, Man, and Cybernetics, 40(6), 2010.

[8] F. Siraj and M.A. Abdoulha. Uncovering hidden information within university's student enrollment data using data mining. In Third Asia International Conference on Modeling and Simulation, 2009. 